\title{
The pneumococcus: carriage, disease and conjugate vaccines
}

\author{
STEVEN OBARO and RICHARD ADEGBOLA* \\ Department of Paediatrics, Imperial College School of Medicine, St Mary's Hospital, Norfolk Place, London \\ W2 1PG, UK and*Medical Research Council Laboratories, PO Box 273, Fajara, The Gambia, West Africa
}

\begin{abstract}
Modern biotechnology has made possible the rapid development and introduction into clinical care of a wide spectrum of potent antimicrobial agents. However, the battle against Streptococcus pneumoniae (pneumococcus) has remained fierce, as acquisition of resistance is even more rapid and these antimicrobial agents are rendered ineffective. Obtaining appropriate antibiotic treatment for severe invasive pneumococcal infections is now a major challenge in many regions of the world. The ground-breaking success of Haemophilus influenzae type b (Hib) conjugate vaccine has brought hope for the conquest of other capsulate bacteria. Recent results of efficacy trials of a heptavalent pneumococcal conjugate vaccine bring hope that protein conjugate vaccines will have a similar impact on pneumococcal disease. These multivalent vaccine formulations include pneumococcal serotypes that most often acquire antibiotic resistance and there is hope that the widespread application of these vaccines will decrease the incidence of multidrug-resistant infections. The potential reduction of pneumococcal disease could even extend to unimmunised younger siblings and the elderly residing with immunised young children, through its herd effect. However, in view of the multiplicity of serotypes and the biology of the pneumococcus, optimism must be tempered by caution.
\end{abstract}

\section{Introduction}

Streptococcus pneumoniae (pneumococcus) is a common commensal of the respiratory epithelium of healthy children and adults [1]. In developing countries, the prevalence of carriage approaches $95 \%$ in healthy children under the age of 3 years and $40 \%$ in adults [2,3]. Carriage of up to four serotypes for several months has been documented, but most pneumococcal infections occur following the recent acquisition of a new serotype $[1,4]$. S. pneumoniae is a common bacterial agent in a wide variety of infections including mucosal infections (e.g., sinusitis and otitis media), pneumonia, arthritis, pericarditis, peritonitis and severe invasive infections such as meningitis and septicaemia [5] (Fig. 1). Mortality due to pneumococcal infections is high, especially in developing countries. World-wide, pneumococcal infections have been estimated to cause 1.2 million pneumonia deaths per year, i.e., nearly $40 \%$ of all pneumonia deaths in children aged $<5$ years [6]. The pneumococcus also

Received 13 June 2001; revised version accepted 5 Sept. 2001.

Corresponding author: Dr S. K. Obaro (e-mail: sobaro@ ic.ac.uk). causes 70000 deaths from meningitis and a similar number of deaths from sepsis and other infections in young children in developing countries each year [6]. In industrialised countries, pneumococcus is the major cause of pneumonia in old age. In the USA 40000 deaths per year are caused by pneumococcal pneumonia or meningitis [7].

The impact of pneumococcal disease is also significant in terms of morbidity. In 1992, the annual incidence of pneumococcal bacteraemic infections in Finland was $24.3 / 100000$ among children up to 4 years of age [8], and that of pneumococcal pneumonia was 11.6 cases/1000 in all age groups [8]. The annual incidence of otitis media episodes among children $<1$ year old in Finland was $0.5-0.7$ episodes per child and during the second year of life $0.5-1.2$ episodes per child [9]. It is estimated that in $43-59 \%$ of culture-verified cases of acute otitis media, pneumococcus is the causative agent [9]. Although a significant proportion of children have pneumococci in their nasopharynx and remain healthy, the mechanisms that promote translation of carriage to disease need to be better understood in order to formulate appropriate interventions. As carriage is often, but not always, an antecedent event in invasive disease and disease transmission is airborne, an inter- 
vention that blocks transmission of pneumococci will greatly reduce the incidence of disease. At present, the World Health Organization (WHO) strategy for controlling pneumococcal disease consists of clinical diagnosis and management with antimicrobial agents. However, this strategy has its limitations. It can help to identify and treat disease but cannot prevent the development of disease. Furthermore, the expertise required for clinical diagnosis is often inadequate or unavailable in many developing countries, where the burden of pneumococcal disease is highest. Moreover, antibiotic resistance among strains of the pneumococcus has become a world-wide problem and it is now a source of major concern. Vaccination holds greater promise for the control of pneumococcal disease through reduction of carriage and transmission. However, the greater number of individual serotypes and their differing biology in the human host makes the battle against the pneumococcus more challenging than that with Haemophilus influenzae type b.

\section{Epidemiology of carriage}

Pneumococci reside in (colonise) the nasopharynx of many individuals but identifiable disease occurs in only a small percentage of persons who are colonised. More than 90 serotypes of $S$. pneumoniae are known but prevalence and serotype pattern vary by geographic location. The mechanisms and epidemiology of carriage, host genetic susceptibility and the onset of invasive disease are still not fully understood.

In a longitudinal carriage study in children in the USA, Gray and colleagues reported that the mean age of acquisition was 6 months [1]. In the first 24 months of life, $95 \%$ of children were colonised at some time and $73 \%$ acquired at least two serotypes (usually on different occasions). Two or three serotypes were present at the same time in $4 \%$ and $0.3 \%$ of specimens, respectively. The duration of carriage was serotypedependent and was commonly between 2.5 and 4.5 months (range 1-17 months). Duration of carriage decreased with successive pneumococcal serotypes and hence was inversely correlated with age [1].

These observations suggest that the rate of pneumococcal carriage correlates with age. Poorly immunogenic serotypes tend to be carried in the nasopharynx of young children for much longer than the more immunogenic serotypes. Also, local antibody production may be important in limiting the duration of carriage. In adults and older children, serotype-specific IgG antibody develops after colonisation and in most cases this occurs in the absence of overt disease. Sequential acquisition of more than one type is common, but infection due to more than one type is rare [1]. It has also been observed that prolonged carriage of single strains is common but infection with that strain is not $[1,10]$. Frequently, the infecting strain has been acquired only recently. Although the age of siblings does not seem to be significant as regards acquisition, children with no siblings tend to acquire their first strain slightly later and acquire fewer types $[1]$.

There are very limited data on longitudinal observations on the carriage of pneumococci in children from developing countries. However, it is generally known that colonisation occurs earlier in life and the prevalence of carriage is much higher than in developed countries. In the Highlands of New Guinea, infants are colonised with pneumococci shortly after birth and most young children are carriers [10]. High pneumococcal carriage rates in children have been recorded in several other developing countries including Zambia [11], Pakistan [12], the Philippines [13] and the Gambia [3, 14]. In Pakistan, high carriage rates were observed in both urban and rural communities. In the Gambia, the carriage rate was highest under the age of 5 years ( $80 \%$ decreasing to $20 \%$ in adulthood) [3]. The decline in carriage rate associated with increasing age may reflect the gradual acquisition of mucosal immunity to the dominant serotypes present in the community but it could also reflect a reduction in exposure. It is not known if carriage of one serotype prevents colonisation by another. In the Gambia, pneumococci of an identical serotype to that responsible for invasive disease in a child were found most frequently among siblings [3], which suggested that they may have been a frequent source of invasive infections in infants. However, the use of more sensitive molecular techniques for genotyping the isolates would be required to confirm this hypothesis.

The pneumococcal nasopharyngeal carriage rates in children in developing countries are generally two-tothree times higher than those found in children from industrialised countries. Crowding, close contacts with a large number of siblings and frequent upper respiratory tract infections are likely to be important risk factors for disease, but it is less clear if they play any role in carriage [15]. High pneumococcal carriage rates are frequent in developing countries and are often associated with carriage of more than one serotype. In the Gambia, $22 \%$ of children carry pneumococci of more than one serotype [16]; in Pakistan [12] the proportion is even higher. The frequency with which multiple carriage occurs is of interest because of the potential for pneumococcal conjugate vaccine to disturb the balance between pneumococci of vaccine and non-vaccine serotypes.

\section{Pathogenesis of pneumococcal disease}

\section{Carriage and disease}

The processes involved in the translocation of the pneumococcus from the nasopharnynx to other sites, including the lung, are probably multifactorial and are 


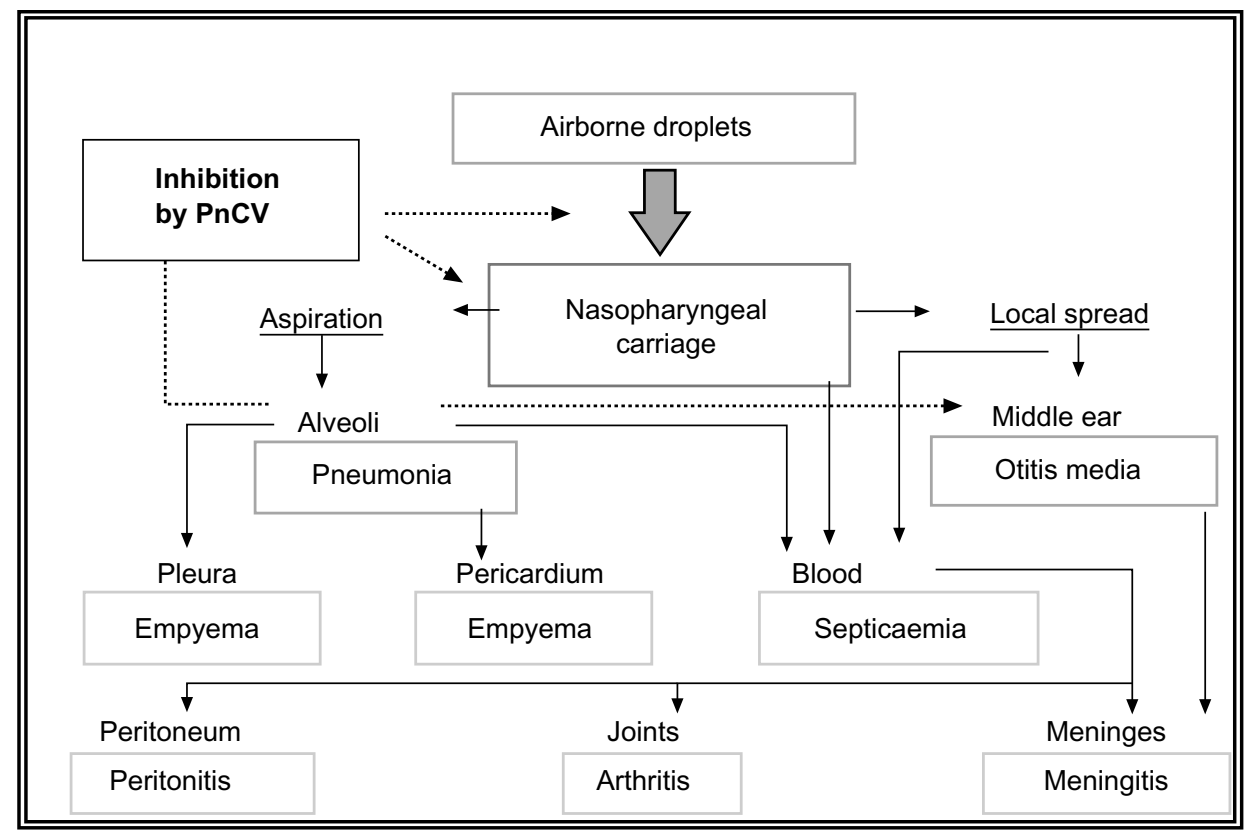

Fig. 1. Pathogenesis of invasive pneumococcal disease.

poorly understood $[17,18]$. It has been generally accepted for several decades that pneumonia results from the aspiration of pneumococci from the upper respiratory tract, although a blood-borne route of dissemination from the upper respiratory tract is also possible [17].

The fact that some pneumococcal serotypes tend to cause disease more frequently in children has led to speculations about the potential role of the immunogenicity of the different serotypes being solely, or at least in part, responsible for this observation. However, it is not clear whether the dominance of these serotypes in carriage is due to their virulence or to other biological characteristics.

Three interchangeable variants of pneumococci, distinguishable by colonial morphology, have been described: opaque, semi-transparent and transparent [19]. These distinct phenotypes have different abilities to colonise the nasopharynx, but the biochemical basis for the phenotypic variation is not known. The bacterium interacts with the glycoconjugate that serves as a receptor on eukaryotic cells in the nasopharynx. Phase variation appears to play a role in the adaptation of pneumococci to changes in the receptors presented on activated host cells [20]. The transparent phenotype appears to possess a selective advantage in the colonisation of the nasopharynx due to its ability to recognise the cognate eukaryotic ligand (G1cNAc $\beta 1$ 3Gal) [21]. More adhesive strains cause localised infections and less adhesive strains cause invasive disease such as bacteraemia and meningitis. Translocation of the organism either by aspiration or penetration of the mucosa results in bacteraemia or sepsis, or both, and seeding in different body systems may cause recognised disease syndromes such as meningitis and arthritis (Fig. 1).

It has been suggested that attachment of the bacteria to the respiratory epithelium is mediated by a disaccharide receptor on fibronectin, present on all epithelial cells [22]. Adherence of pneumococci to tracheal epithelial cells may be enhanced by prior influenza virus infection [23]. This enhancement is probably mediated by viral neuraminidase. This enzyme cleaves sialic acid from glycosphingolipids, structures that are found in substantial amounts in human lung tissue [24]. Thus, neuraminidase is thought to expose other structures that function as receptors for adhering pneumococci.

Investigation into the molecular elements of encounter between host and pathogen suggests that inflammatory activation of human cells shifts the targeting of the pneumococcus to a new receptor, the G-proteincoupled platelet-activating factor (PAF) [25]. Virulent pneumococci engage the PAF receptor. Attachment of the bacterial phosphorylcholine to PAF receptor enhances adherence and invasion of endothelial cells, epithelial cells and PAF receptor-transfected cells. PAF receptor-specific antagonists could arrest this progression in vitro and in vivo [25], thus suggesting a possible novel approach to therapy or prevention of colonisation.

\section{Organisation of host defence}

Defence against pneumococcal infection is dependent upon non-immunological and immunological mechanisms [5, 26-28]. Non-immunological factors include the integrity of the epithelial surface of the upper and 
lower respiratory tract. Abnormalities of this surface appear acutely following viral infection and occur more gradually in tobacco smokers and in persons exposed to air-borne pollutants such as those produced by indoor fires for heating and cooking. In congestive cardiac failure and nephrotic syndrome, circulatory abnormalities leading to pulmonary oedema increase the risk of pneumococcal infection. In acute and chronic alcoholism, depression of the gag reflex, neutropenia and diminished hepatic clearance of opsonised bacteria contribute to the increased risk of pneumococcal disease. Anatomical defects such as a cerebrospinal fluid leakage following skull fracture, which is rare, or an obstruction of the Eustachian tube, which is more common, are risk factors for infection. Metabolic and nutritional abnormalities such as diabetes mellitus and vitamin A deficiency can also be important. At the cellular and molecular level, the exploration of the role of biological response modifiers in the pathogenesis of pneumococcal septicaemia is at an early stage. For example, in experimental animals lethal pneumococcal septicaemia is associated with increased levels of interleukin (IL)-6 [29]. Pre-treatment with the monocyte activator muramyl tripeptide prevents this increase in IL-6, perhaps by desensitising monocytes to later cytokine production, and improves survival.

\section{Mucosal immunity}

The full potential for the control of invasive pneumococcal disease and mucosal infection by intervention at the mucosal level remains to be fully explored. Mucosal immune responses play an important role in the first line of defence against infections with S. pneumoniae [30]. Secretory IgA is thought to be the most important mucosal factor in protection against carriage. However, in contrast to protein antigens, there are limited published data on IgA responses to polysaccharide antigens. Secretory IgA can be detected as early as 6 months, whereas $\mathrm{IgG}$ is rarely detected before 18 months of age. It is interesting that, despite these observations, mucosal immunity to the pneumococcus is relatively immature in young children as compared with adults. Some investigators have reported high numbers of antigen-specific IgA-secreting cells in blood after systemic immunisation of human subjects with polysaccharide antigen [31, 32]. Systemic immunisation without previous exposure to the antigen through the mucosal surface does not usually lead to a secretory $\operatorname{IgA}$ response [32]. Following inhalation, immunological factors such as secretory $\operatorname{IgA}$ or IgG and mechanical factors such as the mucociliary clearance system may prevent colonisation of the nasopharynx.

\section{Laboratory methods}

\section{Isolation of pneumococci from the nasopharynx}

Nasopharyngeal carriage is typically determined by the use of a nasopharyngeal swab, which is inserted into the back of the nasopharynx and then plated on to sheep blood 5\% agar supplemented with gentamicin $5 \mathrm{mg} / \mathrm{L}$ to selectively allow for the optimal growth of $S$. pneumoniae. This has been found to be more sensitive than non-selective media [33]. Recently, a medium containing skimmed milk, tryptone, glucose and glycerol (STGG) has been shown to be a good medium for transport and preservation of pneumococci in nasopharyngeal specimens [34]. However, in areas where direct culture is not possible, antigen detection after enrichment culture may be an alternative method for detection of pneumococcal carriage, but this remains to be explored.

As the swab is applied blindly it is not known how accurately the swabbed sample represents the nasopharyngeal flora. This method of collection has not been formally compared with nasopharnygeal washings. There is evidence to suggest that pneumococci can be cultured more frequently from the nasopharynx than from the oropharynx [35] and it is now standard practice to swab the nasopharynx. A recent study suggests that even well-controlled swab sampling markedly underestimates the rate of pneumococcal carriage. The simultaneous use of quantitative culture and PCR increases the number of positive samples by about one-third, as demonstrated in animal studies [36]. The sensitivity of this method of collection and culture has not been compared with animal inoculation studies. Although animal inoculation studies are labour-intensive and cannot be used for routine nasopharyngeal colonisation studies, such a validation exercise would be invaluable in demonstrating the robustness of the methods of sampling and culture. The quantification of the density of colonisation has also received little attention. Although colony counts have been used as a semi-quantitative measure, the interpretation in terms of disease transmission and pathogenesis may vary for different serotypes and this may be critical for determining the impact of vaccination on the reduction of disease transmission.

\section{Typing of pneumococci}

Suspect pneumococcal isolates are identified by standard morphological, cultural and biochemical characteristics. The standard method for serotype determination has been the Quellung reaction. Other methods such as latex agglutination [37], counterimmuno-electrophoresis [38] and, more recently, flow cytometry [39] have been used. Although several methods are at various stages of development, they need to be standardised against the Quellung reaction. Different investigators evaluate the colonies on the plate differently and the number of colonies picked for capsular typing may influence the number of serotypes identified from the plate. The ability to detect multiple populations of pneumococci from the nasopharyngeal sample remains a critical issue. Current methods, which rely on a culture step before identification of 
serotypes, have limited capacity to detect a minority population of two or more serotypes. Depending on the culture method used, the yield of different serotypes could vary, as the culture method may modify capsule expression if the ambient condition is not optimal for capsule formation. Recently, there have been attempts to improve the detection of minority types by the use of immunoblot assays [40]. However, these methods are labour-intensive and have no definite advantages over the simple latex agglutination test for the detection of multiple serotypes. The goal is an assay that detects multiple populations from nasopharyngeal material without a culture step.

A WHO working group is currently undertaking an international standardisation of serotyping methods. This effort will hopefully culminate in a recommendation for the optimal method for performing carriage studies and in particular, the cultivation and typing of pneumococcal isolates from nasopharyngeal swabs.

\section{Vaccination}

\section{Impact on nasopharyngeal colonisation}

Polyvalent pneumococcal polysaccharide vaccines are licensed for use in adults and children over the age of 2 years who are at high risk for pneumococcal disease. There is an age-dependent response to these vaccines and they are generally poorly immunogenic in children under 2 years of age [41]. Pneumococcal polysaccharide vaccines are T-cell-independent immunogens and are not effective in children under 2 years of age. This is the age group that suffers the highest rates of pneumococcal disease morbidity and mortality. The polysaccharide vaccine does not reduce mucosal carriage of pneumococci, nor does it protect from the related mucosal infections. A landmark in the history of vaccine development was the conjugation of bacterial polysaccharide to carrier proteins, which has been successful for protection against $H$. influenzae type b. By inducing a T-cell-independent immune response, young infants are able to produce antibodies in sufficient quantity to protect against disease. Furthermore, the serum antibody levels are high enough to impact carriage at the mucosal level and this has resulted in the virtual elimination of carriage and, consequently, disease transmission. Conjugate protein technology has been applied to pneumococcal polysaccharides with excellent immunogenicity in children under 2 years of age and promising efficacy results in limited trials for protection against pneumonia, bacteraemia and otitis media in American and Finnish children [41-44].Vaccination offers promising potential for the reduction of carriage of pneumococci and hence transmission. Studies from the Gambia [16], South Africa [45] and Israel [46] have shown a reduction in carriage of vaccine-type pneumococci following vaccination with pneumococcal conjugate vaccines. The observations in South Africa [45] and
Israel [46] were made after three doses of a primary vaccination series, whereas the report from the Gambia [16] was made after a booster dose of pneumococcal polysaccharide vaccine was administered, 18 months after the three-dose primary series. A mathematical model has been applied to the colonisation data from South Africa and the Gambia to ascertain whether the increase in nasopharyngeal carriage of non-vaccine serotypes was due to unmasking or replacement. In both cases, the observed increase was greater than that expected from unmasking alone, thus confirming that true replacement had occurred [47]. Although none of these studies measured secretory antibody concentration, it is apparent that serotype-specific concentrations in serum were achieved that offered protection against carriage of certain vaccine serotypes. It is also possible that the concentration of the secretory antibody that disrupts disease transmission may vary by region, as does the prevalence of carriage and incidence of pneumococcal disease. It is not known whether these vaccines block the acquisition of carriage of a given serotype better than terminating the carriage when the nasopharynx is already colonised. Either of these possibilities could be critical epidemiological determinants in optimising the use of these vaccines.

\section{Impact on antibiotic resistance}

Pneumococci of serotypes 6, 14, 19 and 23 are most notorious for acquiring antibiotic resistance. These are often referred to as paediatric serotypes as they frequently cause a substantial proportion of invasive diseases in children. Some of these are often carried in the nasopharynx of most children and are, therefore, frequently exposed to multiple antibiotics given as prescriptions for minor, largely viral ailments of childhood. Thus, it is not surprising that these pneumococcal serotypes acquire multi-drug resistance quite rapidly. Preliminary data from Israel suggest that a multivalent pneumococcal conjugate vaccine can reduce nasopharyngeal carriage and spread of antibiotic-resistant pneumococci in day-care centres [48]. Thus, there is some hope that the incorporation of these serotypes into the conjugate vaccine formulation may decrease the incidence of multi-drug-resistant infections. However, this is based on the assumption that the alteration of the nasopharyngeal ecology following widespread application of pneumococcal conjugate vaccines does not result in a transfer of resistance genes to the non-vaccine serotypes.

Efficacy trials of the heptavalent pneumococcal conjugate vaccine in the USA have shown a $93 \%$ reduction in invasive pneumococcal disease, $73 \%$ reduction in consolidative pneumonia [43], 7\% reduction in allcause acute otitis media and $20 \%$ reduction in ventilatory tube placements [49]. These are very exciting results that could influence antibiotic prescription practices in regions where the vaccine is in routine use. The threshold for prescribing an antibiotic for a 
febrile child who is fully immunised should be higher than was previously the case. This change in prescription practice will be of enormous importance to daycare centre attendees where the incidence of respiratory infection and the potential for transmission of pneumococci are very high.

\section{Conclusion}

The observation of a reduction in carriage of pneumococcal serotypes incorporated into the conjugate vaccines in three studies was associated with an increase in carriage of non-vaccine serotypes. Whilst it is recognised that protection offered by the pneumococcal conjugate vaccine is serotype-specific, the reduction in carriage of vaccine types may potentially open an ecological niche for the non-vaccine serotypes or indeed for other bacteria. Time will tell what impact this will have on pneumococcal disease, morbidity and mortality. However, other possible explanations must be entertained and evaluated. It is possible that this observation is a consequence of unmasking of minority populations of pneumococci after the 'dominant' vaccine type has been reduced by vaccination. A second and perhaps more disturbing possibility is that the dominant vaccine type has acquired the capsule of non-vaccine type pneumococci by horizontal genetic transfer, and has thus switched its capsule. The evaluation of the real situation will require careful longitudinal studies and would involve combination of several complex molecular techniques [50]. These may include capsular serotyping, antimicrobial susceptibility testing, multilocus enzyme electrophoresis, ribotyping, pulsed-field gel electrophoresis and PCR-based genotyping.

Whether this phenomenon of the alteration in nasopharyngeal flora of pneumococci following vaccination with conjugate pneumococcal vaccine is due to unmasking of the minority, 'non-vaccine type' pneumococci or a capsular switch of the vaccine type pneumococci has practical implications for the widespread use of pneumococcal conjugate vaccines. Recent reports from the Finnish otitis media studies suggest that benefits of protection from otitis media caused by pneumococci of vaccine type may be replaced with disease caused by non-vaccine types following the use of a seven-valent pneumococcal conjugate vaccine [43]. As yet, it is not clear whether a similar phenomenon will be seen for invasive pneumococcal disease.

During this decade, significant progress has been made with the development of protein-based pneumococcal vaccine which hopefully will elicit protective antibodies against all serotypes of pneumoccoci.

In conclusion, there is a need to evaluate further how carriage of pneumococci translates to disease and how this can be controlled. Pneumococcal conjugate vac- cines reduce the carriage of a limited number of pneumococcal serotypes when administered to children, but at the expense of an increase of non-vaccine serotypes, which may be potentially pathogenic. However, the long-term consequence of eliminating major bacterial flora from the nasopharynx will be a cause for concern. Pneumococcal conjugate vaccines have brought high expectations that control of all pneumococcal disease may be achievable, but the limitation on the number of serotypes that can be incorporated in a vaccine formulation suggests that this may not be the panacea for the control of pneumococcal disease. The current tools for determination and evaluation of pneumococci in the nasopharynx need to be refined to capture the complexity of the biology of the pneumococcus and to provide the fundamental knowledge necessary for the optimal control of pneumococcal disease.

\section{References}

1. Gray BM, Converse GM, Dillon HC. Epidemiologic studies of Streptococcus pneumoniae in infants: acquisition, carriage, and infection during the first 24 months of life. J Infect Dis 1980; 142: $923-933$

2. Austrian R. Some aspects of the pneumococcal carrier state. $J$ Antimicrob Chemother 1986; 18 Suppl A: 35-45.

3. Lloyd Evans N, O'Dempsey TJD, Baldeh I et al. Nasopharyngeal carriage of pneumococci in Gambian children and their families. Pediatr Infect Dis $J$ 1996; 15: 866-871.

4. Hansman D. Type distribution and antibiotic sensitivity of Diplococcus pneumoniae. A five year study in Sydney. Med J Aust 1974; 2: 436-440.

5. Musher DM. Infections caused by Streptococcus pneumoniae: clinical spectrum, pathogenesis, immunity, and treatment. Clin Infect Dis 1992; 14: 801-809.

6. Mulholland EK. A report prepared for the Scientific Advisory Group of Experts, Global Programme for Vaccines and Immunization. Geneva, World Health Organization. 1997.

7. Centers for Disease Control. Prevention of pneumococcal disease: recommendation of the Advisory Committee on Immunization Practices (ACIP). MMWR 1997; 46 (No. RR-8).

8. Eskola J, Takala AK, Kela E, Pekkanen E, Kalliokoski R, Leinonen M. Epidemiology of invasive pneumococcal infections in children in Finland. JAMA 1992; 268: 3323-3327.

9. Pukander J, Luotonen J, Sipila M, Timonen M, Karma P. Incidence of acute otitis media. Acta Otolaryngol 1982; 93: 447-453.

10. Gratten M, Montgomery J, Gerega G et al. Multiple colonization of the upper respiratory tract of Papua New Guinea children with Haemophilus influenzae and Streptococcus pneumoniae. Southeast Asian J Trop Med Publ Health 1989; 20: 501-509.

11. Fredericksen B, Hendrichsen J. Throat carriage of Streptococcus pneumoniae and Streptococcus pyogenes among infants and children in Zambia. J Trop Pediatr 1988; 34:114-117.

12. Mastro TD, Nomani NK, Ishaq $Z$ et al. Use of nasopharyngeal isolates of Streptococcus pneumoniae and Haemophilus influenzae from children in Pakistan for the surveillance for antimicrobial resistance. Pediatr Infect Dis J 1993; 12: 824-830.

13. Lankinen KS, Leinonen M, Tupasi TE, Haikala R, Ruutu P. Pneumococci in nasopharyngeal samples from Filipino children with acute respiratory infections. J Clin Microbiol 1994; 32: $2948-2952$.

14. Adegbola RA, Falade AG, Sam BE et al. The etiology of pneumonia in malnourished and well-nourished Gambian children. Pediatr Infect Dis J 1994; 13: 975-982.

15. O'Dempsey TJD, McArdle TF, Morris J et al. A study of risk factors for pneumococcal disease among children in a rural area of west Africa. Int J Epidemiol 1996; 25: 885-893.

16. Obaro SK, Adegbola RA, Banya WAS, Greenwood BM. Carriage of pneumococci after pneumococcal vaccination. Lancet 1996; 348: 271-272. 
17. Busse WW. Pathogenesis and sequelae of respiratory infections. Rev Infect Dis 1991; 13 Suppl 6: S477-S485.

18. Johnston RB. Pathogenesis of pneumococcal pneumonia. Rev Infect Dis 1991; 13 Suppl 6: S509-S517.

19. Weiser JN, Austrian R, Sreenivasan PK, Masure HR. Phase variation in pneumococcal opacity: relationship between colonial morphology and nasopharyngeal colonization. Infect Immun 1994; 62: 2582-2589.

20. Cundell DR, Weiser JN, Shen J, Young A, Tuomanen EI. Relationship between colonial morphology and adherence of Streptococcus pneumoniae. Infect Immun 1995; 63: 757-761.

21. Cundell DR, Tuomanen EI. Receptor specificity of adherence of Streptococcus pneumoniae to human type-II pneumocytes and vascular endothelial cells in vitro. Microb Pathog 1994; 17: 361-374.

22. Andersson B, Dahmén J, Frejd $\mathrm{T}$ et al. Identification of an active disaccharide unit of a glycoconjugate receptor for pneumococci attaching to human pharyngeal epithelial cells. $J$ Exp Med 1983; 158: 559-570.

23. Plotkowski M-C, Puchelle E, Beck G, Jaquot J, Hannoun C Adherence of type 1 Streptococcus pneumoniae to tracheal epithelium of mice infected with influenza A/PR8 virus. Am Rev Respir Dis 1986; 134: 1040-1044.

24. Krivan HC, Roberts DD, Ginsburg V. Many pulmonary pathogenic bacteria bind specifically to the carbohydrate sequence Ga1NAc $\beta 1-4 \mathrm{Gal}$ found in some glycolipids. Proc Natl Acad Sci USA 1988; 85: 6157-6161.

25. Cundell DR, Gerard NP, Gerard C, Idanpaan-Heikkila I, Tuomanen EI. Streptococcus pneumoniae anchor to activated human cells by the receptor for platelet-activating factor. Nature 1995; 377: 435-438.

26. Austrian R. Pneumococcal pneumonia. Diagnostic, epidemiologic, therapeutic and prophylactic considerations. Chest 1986; 90: $738-743$.

27. Gillespie SH. Aspects of pneumococcal infection including bacterial virulence, host response and vaccination. $J \mathrm{Med}$ Microbiol 1989; 28: 237-248.

28. Bruyn GAW, Zegers BJM, van Furth R. Mechanisms of host defense against infection with Streptococcus pneumoniae. Clin Infect Dis 1992; 14: 251-262.

29. Ziegler-Heitbrock HWL, Passlick B, Kafferlein E, Coulie PG, Izbicki JR. Protection against lethal pneumococcal septicemia in pigs associated with decreased levels of IL-6 in blood. Infect Immun 1992; 60: 1692-1694.

30. Reynolds HM. Immunoglobulin $\mathrm{G}$ and its function in the human respiratory tract. Mayo Clin Proc 1988; 63: 161-174.

31. Heilmann C, Barington T, Sigsgaard T. Subclass of individual IgA-secreting human lymphocytes. Investigation of in vivo pneumococcal polysaccharide-induced and in vitro mitogeninduced blood B cells by monolayer plaque-forming cell assays. J Immunol 1988; 140: 1496-1499.

32. Tarkowski A, Lue C, Moldoveanu H, Kiyono H, McGhee JR, Mestecky J. Immunization of humans with polysaccharide vaccines induces systemic, predominantly polymeric IgA2subclass antibody responses. J Immunol 1990; 144: 3770-3778.

33. Converse GM, Dillon HC. Epidemiological studies of Streptococcus pneumoniae in infants: methods of isolating pneumococci. J Clin Microbiol 1977; 5: 293-296.

34. O'Brien KL, Bronsdon MA, Dagan $\mathrm{R}$ et al. Evaluation of a medium (STGG) for transport and optimal recovery of Streptococcus pneumoniae from nasopharyngeal secretions collected during field studies. J Clin Microbiol 2001; 39: 1021-1024.

35. Rapola S, Salo E, Kiiski P, Leinonen M, Takala AK. Comparison of four different sampling methods for detecting pharyngeal carriage of Streptococcus pneumoniae and Haemophilus influ- enzae in children. J Clin Microbiol 1997; 35: 1077-1079.

36. Kontiokari T, Renko M, Kaijalainen T, Kuisma L, Leinonen M. Comparison of nasal swab culture, quantitative culture of nasal mucosal tissue and PCR in detecting Streptococcus pneumoniae carriage in rats. APMIS 2000; 108: 734-738.

37. Lafong AC, Crothers E. Simple latex agglutination method for typing pneumococci. J Clin Pathol 1988; 41: 230-231.

38. Holliday MG. Serotyping of pneumococci by polyvalent counter- immunoelectrophoresis. $J$ Hosp Infect 1985; 6: $110-111$.

39. Park MK, Briles DE, Nahm MH. A latex bead-based flow cytometric immunoassay capable of simultaneous typing of multiple pneumococcal serotypes (Multibead assay). Clin Diagn Lab Immunol 2000; 7: 486-489.

40. Bronsdon MA, O'Brien KL, Facklam RR, Schwartz B, Carlone GM. Immunoblot detection of multiple serotypes of Streptococcus pneumoniae (Pnc) in naropharyngeal (NP) specimens using monoclonal antibodies (MAb). The Second International Symposium on Pneumococci and Pneumococcal Disease, Sun City, South Africa. 19-23 March, 2000. Abstract P96.

41. Eskola J. Immunogenicity of pneumococcal conjugate vaccines. Pediatr Infect Dis J 2000; 19: 388-393.

42. Rennels MB, Edwards KM, Keyserling HL et al. Safety and immunogenicity of heptavalent pneumococcal vaccine conjugated to CRM197 in United States infants. Pediatrics 1998; 101: 604-611.

43. Black S, Shinefield H, Fireman B et al. Efficacy, safety and immunogenicity of heptavalent pneumococcal conjugate vaccine in children. Northern California Kaiser Permanente Vaccine Study Center Group. Pediatr Infect Dis J 2000; 19: 187-195.

44. Black S, Shinefield H, Ray $P$ et al. Efficacy of heptavalent conjugate pneumococcal vaccine (Wyeth Lederle) in 37,000 infants and children: impact on pneumonia, otitis media and an update on invasive disease - Results: extended follow-up of the efficacy trial cohort. Second International Symposium on Pneumococi and Pneumococcal Disease, Sun City, South Africa. 19-23 March 2000. Abstract O19.

45. Mbelle N, Huebner RE, Wasas AD, Kimura A, Chang I, Klugman KP. Immunogenicity and impact on nasopharyngeal carriage of a nonavalent pneumococcal conjugate vaccine. $J$ Infect Dis 1999; 180: 1171-1176.

46. Dagan R, Muallem M, Melamed R, Leroy O, Yagupsky P. Reduction of pneumococcal nasopharyngeal carriage in early infancy after immunization with tetravalent pneumococcal vaccines conjugated to either tetanus toxoid or diphtheria toxoid. Pediatr Infect Dis J 1997; 16: 1060-1064.

47. Lipsitch M. Bacterial vaccines and serotype replacement: lessons from Haemophilus influenzae and prospects for Streptococcus pneumoniae. Emerg Infect Dis 1999; 5: 336-345.

48. Dagan R, Givon-Lavi N, Porat N, Sikuler-Cohen M, Fraser D. Immunization of toddlers attending day care centers (DCC) with a 9-valent conjugate pneumococcal vaccine (PncCRM9) reduces transmission of Streptococcus pneumoniae (Pnc) and antibiotic resistant $S$. pneumoniae (R-Pnc) to their young siblings. 40th Interscience Conference on Antimicrobial Agents and Chemotherapy. 17-20 September 2000. Toronto, Ontario, Canada. Abstract 687.

49. Kilpi T, Jokinen J, Herva E and the Finnish Otitis Media (FinOM) Study Group. Effect of a heptavalent pneumococcal conjugate vaccine on pneumococcal acute otitis media by serotype. Second International Symposium on Pneumococci and Pneumococcal disease, Sun City, South Africa. 19-23 March 2000. Abstract O20.

50. Spratt GBG, Greenwood BM. Prevention of pneumococcal disease by vaccination: does serotype replacement matter? Lancet 2000; 356: 1210-1211. 